\title{
Monoenergetic proton beam accelerated by single reflection mechanism only during hole-boring stage
}

\author{
Wenpeng Wang ${ }^{1, *}$, Cheng Jiang ${ }^{1}$, Shasha $\mathrm{Li}^{1}$, Hao Dong ${ }^{1}$, Baifei Shen ${ }^{2}$, Yuxin Leng ${ }^{1}$, Ruxin $\mathrm{Li}^{1}$, \\ and Zhizhan $\mathrm{Xu}^{1}$ \\ ${ }^{1}$ State Key Laboratory of High Field Laser Physics, Shanghai Institute of Optics and Fine Mechanics, Chinese Academy of Sciences, \\ Shanghai 201800, China \\ ${ }^{2}$ Department of Physics, Shanghai Normal University, Shanghai 200234, China \\ (Received 9 May 2019; revised 15 June 2019; accepted 12 July 2019)
}

\begin{abstract}
Multidimensional instabilities always develop with time during the process of radiation pressure acceleration, and are detrimental to the generation of monoenergetic proton beams. In this paper, a sharp-front laser is proposed to irradiate a triple-layer target (the proton layer is set between two carbon ion layers) and studied in theory and simulations. It is found that the thin proton layer can be accelerated once to hundreds of $\mathrm{MeV}$ with monoenergetic spectra only during the hole-boring (HB) stage. The carbon ions move behind the proton layer in the light-sail (LS) stage, which can shield any further interaction between the rear part of the laser and the proton layer. In this way, proton beam instabilities can be reduced to a certain extent during the entire acceleration process. It is hoped such a mechanism can provide a feasible way to improve the beam quality for proton therapy and other applications.
\end{abstract}

Keywords: proton acceleration; radiation acceleration; sharp-front laser; hole-boring stage; light-sail stage

\section{Introduction}

With the development of laser technology ${ }^{[1-4]}$, laser-driven ion beams have attracted much attention owing to potential applications such as fast ignition of inertial confinement fusion $^{[5,6]}$, medical therapy ${ }^{[7-9]}$, proton imaging ${ }^{[10]}$, neutron production $^{[11,12]}$, nuclear physics ${ }^{[13,14]}$ and pre-accelerators for conventional acceleration devices ${ }^{[15]}$.

Radiation pressure acceleration $(\mathrm{RPA})^{[16-30]}$ is usually considered as an efficient mechanism to accelerate the whole target to gigaelectronvolts through the 'holeboring' (HB) ${ }^{[16,31]}$ and 'light-sail' (LS) ${ }^{[18,20,21,32]}$ stages. However, the beam spectra in RPA experiments ${ }^{[22,26,29]}$ are much worse than what the theoretical results indicate $^{[16-21,23-25,27,28]}$. One possible reason is the presence of multidimensional instabilities, such as RayleighTaylor-like (RT-like) instability ${ }^{[19,20,33-36]}$ and Weibellike instability ${ }^{[37,38]}$. Recent studies have given accurate predictions of the mode structures of the instabilities and the growth rates for a wide range of laser and plasma

Correspondence to: W. Wang, Shanghai Institute of Optics and Fine Mechanics, Chinese Academy of Sciences, Shanghai 201800, China. Email: wangwenpeng@siom.ac.cn

* This article has been amended since original publication to correct the first author's name. parameters $^{[27]}$. These show that the surface ripples are more likely induced by coupling between the transverse oscillating electrons and the quasistatic ions near the laserplasma interface, although the target surface is initially flat. It indicates that instabilities are intrinsically generated as the laser irradiates the interface of the plasma. Previously, a model driven by the front or flat-top parts of the laser has been investigated ${ }^{[27]}$. However, in real cases, the rear part of the laser pulse may further disturb the particle beam in RPA because it continues to interact with the laser-plasma interface. In addition, the accelerating gradient becomes lower in the LS stage, where the charge separation field is reduced due to the Doppler effects of the flying target on the driven laser, which is detrimental to controlling development of multidimensional instabilities in the relativistic region.

In this paper, the proton beam is prevented from moving together with the laser-plasma interface during the entire acceleration process, which may intrinsically reduce the development of detrimental instabilities. Here, a single reflection mechanism is used to stably accelerate the proton beam by optimally designing the multilayer target (the proton layer is set between two carbon ion layers). Such a multilayer target is totally different from previous cases ${ }^{[39,40]}$, where the heavy ion layer is set between the proton layers. There, the heavy ions in the middle are accelerated together with protons at a lower velocity in a collisionless shock 
acceleration manner. However, in our case, the middle proton layer is separated from the heavy ions by means of a sharp-front laser, which is reflected once to hundreds of $\mathrm{MeV}$ with a monoenergetic spectrum only during the HB stage. Hence, the proton beam has a greatly reduced chance of moving together with the laser-plasma interface during the entire acceleration process. In this way, some multidimensional instabilities can be reduced to a certain extent. It could potentially be used to improve the beam quality for proton therapy and other applications.

\section{Model and simulation}

First, we review the traditional RPA process in a onedimensional simulation to help us to design the target in the single reflection mechanism (SRM). A circularly polarized laser arrives at the target at $t=20 T$ (see Figure 1(a)), where $T=\lambda / c$ and $\lambda=1 \mu \mathrm{m}$ is the laser wavelength. $c$ is the speed of light in vacuum. In the simulation, the laser amplitude has a triangular profile in time (linear upramp $t_{\text {up }}=2.2 T$ and linear down-ramp $t_{\text {down }}=2.2 T$ ). The pressure of the circularly polarized laser stably pushes electrons forward, such that the electrons are piled up at the front of the laser, forming a compressed electron layer. Previous simulations have demonstrated that the velocity of such a compressed electron layer is uniform because the laser has a linear front ${ }^{[24,41]}$. In this case, the velocity of such an electron layer $\left(v_{\mathrm{CEL}}\right)$ can be calculated from the balance between the laser pressure force $\left(2 a^{2}\left(1-v_{\mathrm{CEL}}\right) /\left(1+v_{\mathrm{CEL}}\right)\right)$ and electrostatic force $\left(2\left(\pi n_{0} v_{\mathrm{CEL}} t\right)^{2}\right)$. In the following calculations, the length, time, velocity, density, charge and field are normalized by $\lambda, \lambda / c, c, \omega_{\mathrm{L}}^{2} m_{\mathrm{e}} / 4 \pi \mathrm{e}^{2},-e$ and $e / m_{\mathrm{e}} \omega_{\mathrm{L}} c$, respectively, for the theoretical compact calculation:

$$
\begin{aligned}
2 a^{2}\left(1-v_{\mathrm{CEL}}\right) /\left(1+v_{\mathrm{CEL}}\right)=2\left(\pi n_{0} v_{\mathrm{CEL}} t\right)^{2} & \\
v_{\mathrm{CEL}}= & \frac{1-3 \kappa^{2}}{3+3 \kappa^{2}}+\sqrt[3]{M+\sqrt{M^{2}+N^{3}}} \\
& +\sqrt[3]{M-\sqrt{M^{2}+N^{3}}},
\end{aligned}
$$

where $M=-\left(27 \kappa^{4}-36 \kappa^{2}+1\right) /\left[27\left(1+\kappa^{2}\right)^{3}\right], N=$ $\left(15 \kappa^{2}-1\right) /\left[9\left(1+\kappa^{2}\right)^{2}\right]$ and $\kappa=a_{0} / \pi t_{\text {up }} n_{0}$. Here $a_{0}=$ $e E_{\mathrm{L}} / m_{\mathrm{e}} \omega_{\mathrm{L}} c \approx 94$ is the dimensionless peak amplitude of laser, $E_{\mathrm{L}}$ is the amplitude of the laser electric field, $\omega_{\mathrm{L}}$ is the laser frequency, and $m_{\mathrm{e}}$ and $e$ are the electron rest mass and charge, respectively. $a=\left(a_{0} / t_{\text {up }}\right)\left(t-v_{\text {CEL }} t\right)$ is the normalized laser amplitude at the electron layer. The foil density is $n_{0}=50 n_{\mathrm{c}}$ and the foil thickness is $d=$ $0.5 \mu \mathrm{m}$. Then $v_{\mathrm{CEL}} \sim 0.183 c$ can be obtained according to Equation (2). Here an idealized triangular laser profile is used in the present scheme, mainly for simplicity of the calculation and simulation. For the case of a Gaussian temporal profile, the velocity of the compressed electron
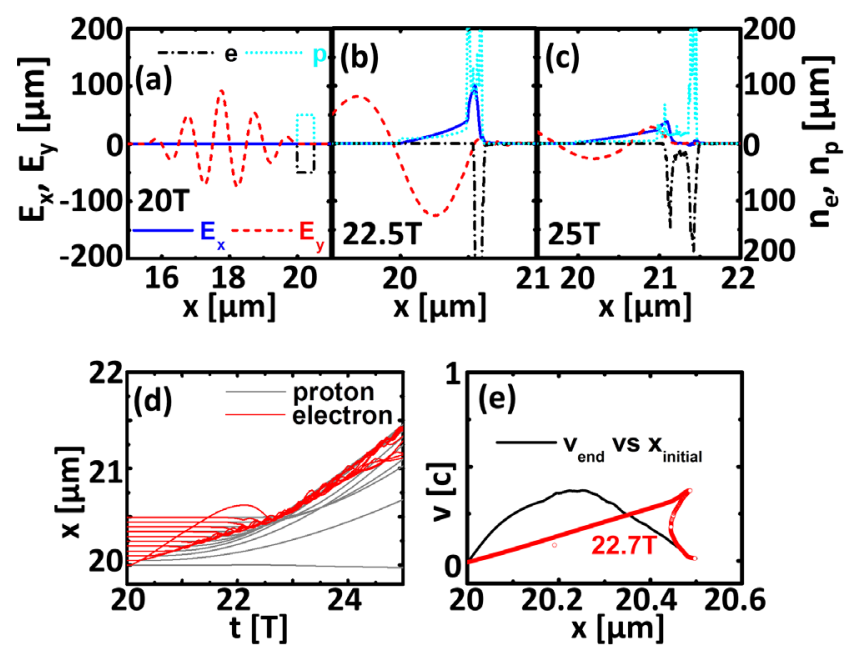

Figure 1. Electric field $E_{x}$ (blue solid line) and $E_{y}$ (red dash line), electron density (black dash-dot line), proton density (cyan dot line) at (a) $t=20 T$, (b) $t=22.5 T$ and (c) $t=25 T$. (d) Trajectories of electrons (red solid line) and protons (gray solid line) in the simulations. (e) Phase space distributions of protons at $t=22.7 T$ (red circles). The black solid line represents the velocity distribution at the end of the $\mathrm{HB}$ stage for protons initially at different positions of the foil ( $v_{\text {end }}$ versus $\left.x_{\text {initial }}\right)$.

layer is no longer uniform, and the calculation becomes complex, but can be solved by the time-dependent model used in our previous work ${ }^{[42]}$.

Initially, the protons lag behind the compressed electron layer because the proton mass $m_{\mathrm{i}}=1836 m_{\mathrm{e}}$ is much greater than the electron mass. At the end of HB stage, the fastest protons reach the compressed electron layer (see Figures 1(b) and 1(d) ) ${ }^{[24]}$ and the LS stage starts. It should be noted that the protons initially in the target center are accelerated faster than the others at the end of the HB stage in this case ${ }^{[24]}$. The velocities of these protons are mainly distributed around $0.4 c$ from $x_{\text {initial }}=20.2 \mu \mathrm{m}$ to $20.25 \mu \mathrm{m}$ (see Figure 1(e)). It is also noted that a narrow energetic spectrum may be obtained if we selectively accelerate the protons in the middle layer. From Figure 1(e), it can also be seen that the spectrum of the middle proton layer becomes broadened with an increased thickness of the middle proton layer, so a thinner middle proton layer will be better.

Then, the multilayer target is designed to ensure that the middle proton layer can be selectively accelerated. According to Figure 1(e), a proton layer from $x=20.2 \mu \mathrm{m}$ to $x=20.25 \mu \mathrm{m}$ is set between the carbon $\left(\mathrm{C}^{6+}\right)$ layers (corresponding regions are $20 \mu \mathrm{m}<x<20.2 \mu \mathrm{m}$ and $20.25 \mu \mathrm{m}<x<20.5 \mu \mathrm{m})$. The electron density is $n_{0}=$ $50 n_{\mathrm{c}}$ and the densities of the proton and $\mathrm{C}^{6+}$ layers are $n_{\mathrm{p}}=50 n_{\mathrm{c}}$ and $n_{\mathrm{C} 6+}=8.3 n_{\mathrm{c}}$, respectively. Here, the chargeto-mass ratio of $\mathrm{C}^{6+}$ is $1 / 2$, which is half of the value for the proton (that is, 1), meaning that protons can be more easily accelerated compared with $\mathrm{C}^{6+}$ ions. So, the $\mathrm{C}^{6+}$ ions in the rear part of the target can be assumed to be at rest when the proton layer arrives. Different from the cases depicted 
in Figure 1, the trajectories of the protons initially at the middle of the target will cross trajectories of the $\mathrm{C}^{6+}$ ions initially at the rear of the target. Hence, the protons can be accelerated by the charge separation field $E_{x}=E_{0} x(t) / d$, where $E_{0}$ is the maximum charge separation field, given by $E_{0}=4 \pi e n_{0} d$. The proton velocity can be calculated using the following equation:

$$
v_{\mathrm{p}}(t+\mathrm{d} t)=\frac{4 \pi^{2} n_{0} x(t)}{m_{\mathrm{p}} \gamma_{\mathrm{p}}(t)} \mathrm{d} t+v_{\mathrm{p}}(t)
$$

where $m_{\mathrm{p}}=1836$ is the proton mass and $\gamma_{\mathrm{p}}(t)=$ $1 / \sqrt{1-v_{\mathrm{p}}^{2}(t)}$ is the relativistic factor for the protons. In addition, the position of the protons can be calculated using the following equation:

$$
x_{\mathrm{p}}(t+\mathrm{d} t)=\frac{2 \pi^{2} n_{0} x(t)}{m_{\mathrm{p}} \gamma_{\mathrm{p}}(t)}(\mathrm{d} t)^{2}+v_{\mathrm{p}}(t) \mathrm{d} t+x_{\mathrm{p}}(t) .
$$

The dynamics of the middle proton layer can be obtained from Equations (3) and (4) for $v_{\mathrm{p}}(t=20 T)=0$ and $x_{\text {initial }}=$ $20 \mu \mathrm{m}+0.5 d$. It should be noted that all these equations are related to the electron density $n_{0}$, and the dynamics of the different ions are determined by their charge-to-mass ratios. The accelerating scheme will be totally different if their densities change.

Based on Equations (3) and (4), the middle proton layer arrives at the back surface of the target $\left(x_{\text {initial }}=20.5 \mu \mathrm{m}\right)$ at $t \sim 22.6 T$. To start the LS stage as soon as possible, the compressed electron layer should also arrive at the back of the target simultaneously with the middle proton layer, forming a double layer. So, the velocity of the compressed electron layer should be $v_{\mathrm{CEL}}=d / 2.6 T \sim 0.2 c$. For the linearly rising-up laser front, the velocity $v_{\mathrm{CEL}}$ during the HB stage can be calculated according to Equations (1) and (2). Considering $a=\left(a_{0} / t_{\mathrm{up}}\right)\left(1-v_{\mathrm{CEL}}\right) t$, the steepness of the laser front can be obtained as

$$
a_{0} / t_{\mathrm{up}}=\pi n_{0} v_{\mathrm{CEL}}\left(1+v_{\mathrm{CEL}}\right)^{1 / 2}\left(1-v_{\mathrm{CEL}}\right)^{-3 / 2} .
$$

Then, $a_{0} / t_{\text {up }} \sim 48$ is obtained for $n_{0}=50 n_{\mathrm{c}}$ and $v_{\mathrm{CEL}} \sim$ $0.2 c$, as shown in Figure 2(a). The peak amplitude $a_{0}$ of the laser can be calculated as $\sim 96$, according to $a_{0}=$ $\left(a_{0} / t_{\text {up }}\right)\left(1-v_{\mathrm{CEL}}\right) d / v_{\mathrm{CEL}}$ and $t_{\text {up }} \sim 2 T$. Here, the velocity of the proton layer can increase up to $v_{\mathrm{p}} \sim 0.4 c$, which is almost twice the velocity $v_{\mathrm{CEL}}(\sim 0.2 c)$. This indicates that the proton layer moves faster than the compressed layer. At the same time, the remaining electrons will move together with the other $\mathrm{C}^{6+}$ ions, because the laser intensity begins to decrease after $t \sim 22.6 T$. The velocity of the $\mathrm{C}^{6+}$ electron double layer is lower than the velocity of the protonelectron double layer. So, the rear part of the laser can be reflected by the $\mathrm{C}^{6+}$-electron double layer. Only the carbon ions are heated and spread extensively, reducing the multidimensional instabilities of the proton layer to a certain

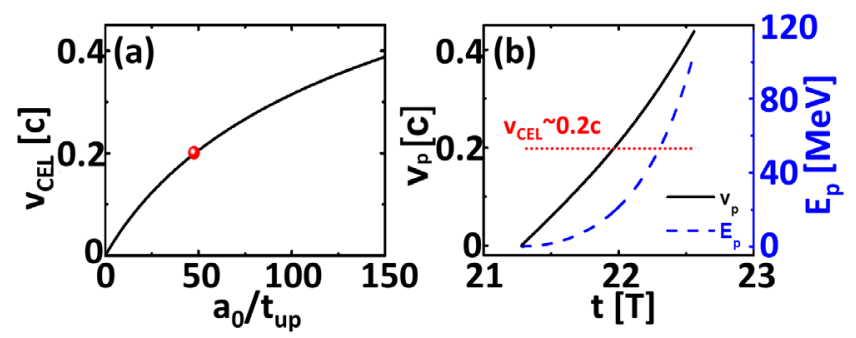

Figure 2. (a) Relation between the velocity of the compressed electron layer $v_{\mathrm{CEL}}$ and the steepness of the laser front $a_{0} / t_{\mathrm{up}}$ according to Equation (5) for $n_{0}=50 n_{\mathrm{c}}$. (b) Evolutions of the velocity, $v_{\mathrm{p}}$ (black solid line), and energy, $E_{\mathrm{p}}$, of the proton layer during the HB stage.

extent. Ultimately, the high-quality proton bunch can be selectively accelerated to $E_{\mathrm{p}} \sim 100 \mathrm{MeV}$ at the end of the HB stage (see Figure 2(b)). It is believed that the single reflection mechanism can be realized only during the HB stage according to Equations (3)-(5). It should be noted that Equations (3)-(5) cannot be applied for the special case $t_{\text {up }}=0 T$, which should be specially solved by the timedependent models ${ }^{[42]}$.

Two-dimensional particle-in-cell simulations are carried out to verify the theoretical expectations of the single reflection mechanism. A multilayer target is designed as shown in Figure 3. The hydrogen layer lies in the middle $(20.2 \mu \mathrm{m} \leqslant x \leqslant 20.25 \mu \mathrm{m})$ of the foil. The carbon layer lies in the regions $20 \mu \mathrm{m}<x<20.2 \mu \mathrm{m}$ and $20.25 \mu \mathrm{m}<x<$ $20.5 \mu \mathrm{m}$. The hydrogen and carbon atoms are assumed to be ionized to $\mathrm{H}^{+}$and $\mathrm{C}^{6+}$ before the main pulse is incident on the target. The electron density is $n_{0}=50 n_{\mathrm{c}}$. The densities of the $\mathrm{H}^{+}$and $\mathrm{C}^{6+}$ layers are $n_{\mathrm{p}}=50 n_{\mathrm{c}}$ and $n_{\mathrm{C} 6+}=8.3 n_{\mathrm{c}}$, respectively. The laser amplitude has a triangular profile in time (linear up-ramp $t_{\text {up }}=2 T$ and linear down-ramp $t_{\text {down }}=$ $2 T$ ) with a peak value $a_{0}=96$. The pulse waist is $10 \mu \mathrm{m}$ (FWHM). The simulation box size is $50 \mu \mathrm{m}(x) \times 60 \mu \mathrm{m}(y)$, and the number of spatial grids is $8000 \times 9600$. Each is filled with 20 macroelectrons and 20 macroprotons (or $\mathrm{C}^{6+}$ ions).

Figure 3 depicts the detailed progress from the $\mathrm{HB}$ to the LS stage as the CP laser irradiates the multilayer target. Initially, the electrons are stably pushed forward because there are no oscillating terms in the expression for the ponderomotive force for CP lasers ${ }^{[19]}$, as shown in Figure 3(d). The $\mathrm{C}^{6+}$ ions lag behind the compressed electron layer initially. The charge separation field, $E_{x}$, then becomes stronger with the increased distance between the electrons and the ions. Both $\mathrm{C}^{6+}$ ions and protons begin to be accelerated by $E_{x}$. At $t=23 T$, the compressed electron layer has reached the back of the target together with the proton layer, which is almost consistent with the theoretical assumption ( $t \sim 22.6 T)$ according to Equation (4) (refer to Figures 3(e) and 3(k)). Based on Equation (1), the velocity of the proton layer $\left(v_{\mathrm{p}} \sim 0.4 c\right)$ is much higher than the velocity of the compressed electron layer $\left(v_{\mathrm{CEL}}=\sim 0.2 c\right)$. This means the proton layer is accelerated and separated 


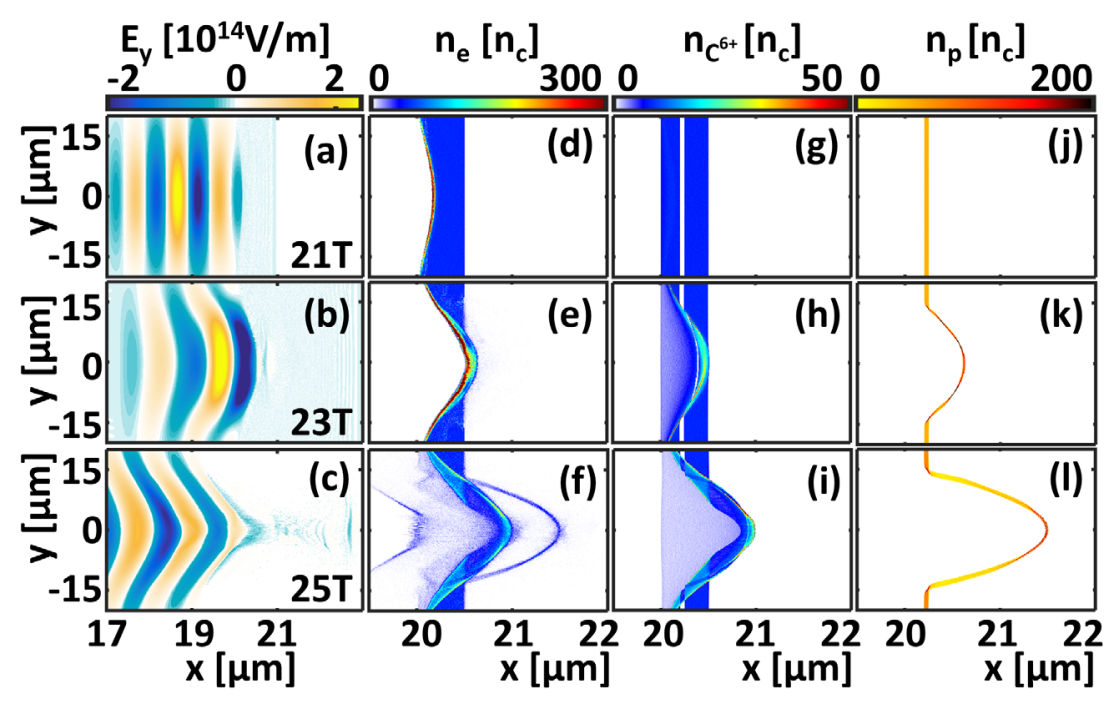

Figure 3. Distributions of (a)-(c) electric field $E_{y}$, (d)-(f) electron density $n_{\mathrm{e}},(\mathrm{g})-(\mathrm{i}) \mathrm{C}^{6+}$ density and (j)-(l) proton density at $t=21 T$ (first row), $t=23 T$ (second row) and $t=25 T$ (third row).
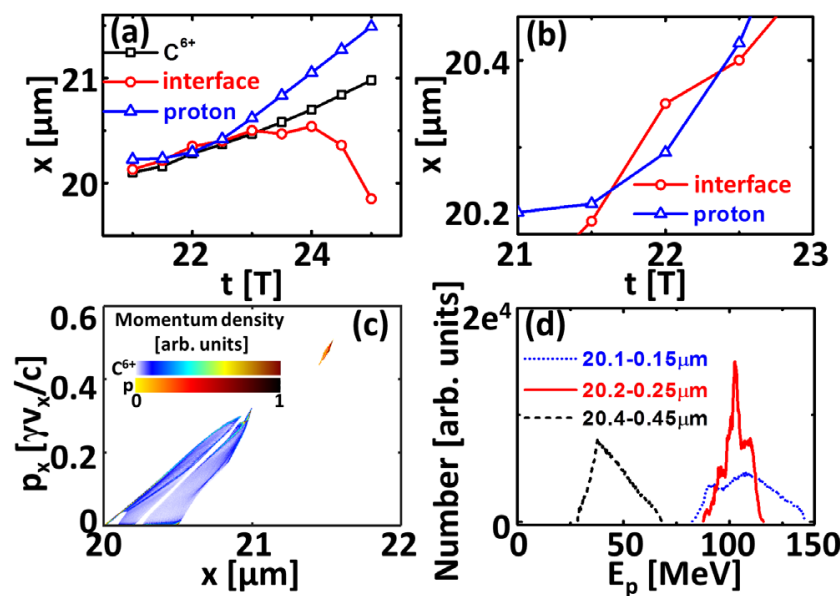

Figure 4. (a) Trajectories of the $\mathrm{C}^{6+}$ layer (black square), the proton layer (blue triangle) and the interface between the laser and the compressed electron layer (red circle). Enlarged plots of the trajectories are shown in (b). (c) Phase space distributions of $\mathrm{C}^{6+}$ ions and protons at $t=25 T$. (d) Energetic spectra for the proton layer in different initial regions at $t=25 T$. Here, protons in the region $-3 \mu \mathrm{m}<y<3 \mu \mathrm{m}$ are considered.

from the compressed electron layer in the LS stage. In addition, the peak decreases after $t=22.6 T$. To maintain the balance between the laser pressure and charge separation forces again, the charge separation field, $E_{x}$, weakens. This is realized by the $\mathrm{C}^{6+}$ ions reaching the compressed electron layer after $t=22.6 T$. Finally, two double layers are formed (see Figures 3(f)-3(l)). The $\mathrm{C}^{6+}$-electron layer is heated and spreads extensively in space until the laser is totally reflected at $t=25 T$ (see Figure 3(c)). In contrast, the protonelectron layer always moves ahead of the $\mathrm{C}^{6+}$-electron layer, maintaining a compact high-quality bunch, which verifies the theoretical model (Equations (3)-(5)).
It should be noted that the proton layer has a greatly reduced probability of moving together with the laser-plasma interface in this case, as can clearly be seen from Figures 4(a) and 4(b). Initially, the laser interface moves forward and overtakes the proton layer at $t \sim 21.5 T$. At the end of the HB stage $(t \sim 22.5 T)$, the proton layer overtakes the interface. After $t \sim 22.5 T$, the proton layer continues to move ahead of both the interface and $\mathrm{C}^{6+}$ ions with a constant velocity, because the laser pulse is reflected by the $\mathrm{C}^{6+}$-electron double layer. As depicted in Figure 4(a), the laser pulse is completely reflected away from the $\mathrm{C}^{6+}$ layer, and does not affect the proton layer. Here, the proton layer is just 'slingshot-likely' reflected once by the charge separation field only during the HB stage (refer to Figure 4(c)). Thus, instabilities of the laser-plasma interface are intrinsically reduced. A pure proton beam with a quasimonoenergetic spectrum $(\sim 5 \%)$ centered at $\sim 100 \mathrm{MeV}$ is finally generated at $t=25 T$ (see Figure 4(d)). From Figures 4(a) and 4(b), it can be found that the proton layer is separated from the carbon layers, and the carbon layer blocks the rear parts of the laser after $t \sim 23 T$, so the spectrum of the proton beam does not change much after $t \sim 23 T$.

The accelerations of the proton layers in the different regions are compared to verify the optimum conditions for the single reflection mechanism, shown in Figure 4(c). The center energy of the proton layer $\left(20.4 \mu \mathrm{m}<x_{\text {initial }}<\right.$ $20.45 \mu \mathrm{m}$ ) is only $E_{\mathrm{p}}=39 \mathrm{MeV}$. The main reason is that the proton layer, initially at rear part of the foil, is accelerated for a shorter time during the HB stage. In contrast, the proton layer initially at the front can be accelerated for a longer time to a higher maximum energy $(137 \mathrm{MeV})$. However, the spectrum spread $(\sim 35 \%)$ is much worse than the optimum case $(\sim 5 \%)$. On the one hand, this is caused by the disturbed charge separation field when the falling part of the laser 


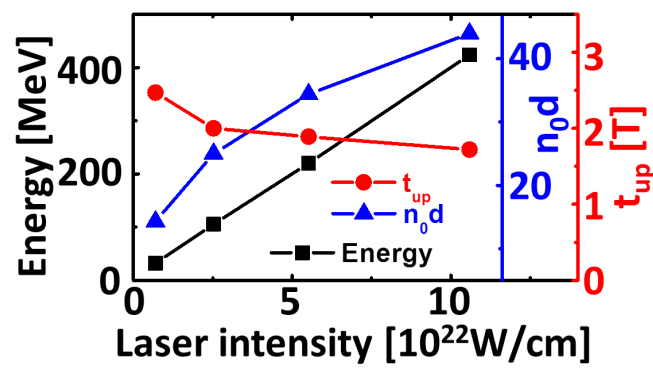

Figure 5. Rising-up duration of the laser front $t_{\text {up }}$ (red circle), energy (black square) and areal density (blue triangle) of the proton layer for different laser intensities calculated from Equations (3)-(5). Here the foil density is $n_{0}=50 n_{\mathrm{c}}$.

pulse is reflected. On the other hand, the spectrum spread is intrinsically best for the proton layer in the middle layer, as shown in Figure 1(e).

\section{Discussion}

In the future, the development of $10 \mathrm{PW}$, and even $100 \mathrm{PW}$, laser systems ${ }^{[43-46]}$, and target fabrication ${ }^{[47,48]}$ could lead to reaching laser intensities of the order of $10^{22}-10^{23} \mathrm{~W} / \mathrm{cm}^{2}$, which can easily accelerate the proton layer to energies of hundreds of $\mathrm{MeV}$ (refer to Figure 5). For example, $\sim 400 \mathrm{MeV}$ protons can be generated by a $\sim 10 \mathrm{fs,}$ $250 \mathrm{~J}$ laser $(\sim 50 \%$ energy in a $2-\mu \mathrm{m}$ focal spot (FWHM), corresponding to a laser intensity of $\sim 1 \times 10^{23} \mathrm{~W} / \mathrm{cm}^{2}$ ) irradiating the multilayer foil (areal density $\sim 44 n_{\mathrm{c}} \lambda$ ). In proton cancer therapy, monoenergetic proton beams with a tunable energy of 50-250 MeV are required to target tumor locations, which can be realized by the single reflection mechanism proposed in this paper. It should be noted that optical components based on plasmas can provide a solution to the manipulation of the polarization state of the sharpfront laser ${ }^{[49]}$, as well as its temporal profile ${ }^{[26,50,51]}$. In fact, Equations (3)-(5) are general, helping us to find other optimum parameters of the laser and target under the present lab conditions.

It should be noted that the efficiency of proton acceleration only in the HB stage seems lower, compared to the previous PRA process including the LS stage ${ }^{[18,20,21,32]}$. In previous works, the laser interacts with the proton beam for a longer time, so that a higher beam energy $(\mathrm{GeV}$, even $10 \mathrm{GeV})$ and higher accelerating efficiency can be obtained. Especially in the LS stage, the efficiency can be near $100 \%$. However, such good acceleration does not occur in realistic experiments. The main reason for this is that serious multidimensional instabilities develop during the laser-plasma interaction. These instabilities may arise due to intrinsic turbulence as the laser irradiates the interface of the plasma, or the unpredictable irregular shapes of the target surface and laser front. So the maximum energy of the proton beam is only $\sim 100 \mathrm{MeV}$ in the present experiments. Also, the spectra are not as good as what the simulations predict. In this paper, we wanted simply to use a sharp-front laser irradiating a triple-layer target to reduce the interaction time between the laser and the proton beam. In this way, the instabilities can be reduced to a certain extent, although the acceleration efficiency is lower. It is believed that proton beams with monoenergetic spectra of hundreds of $\mathrm{MeV}$ can potentially be applied in proton therapy.

\section{Conclusion}

In conclusion, a proton layer with pure spectra can be successfully accelerated once to hundreds of $\mathrm{MeV}$ only during the HB stage. The proton beam has a reduced probability of moving together with the laser-plasma interface during the entire acceleration process. In this manner, some multidimensional instabilities, such as Rayleigh-Taylor-like instability and Weibel-like instability, can be reduced to a certain extent. This provides a feasible method to realize proton therapy and other applications using multi-PW laser system in the future.

\section{Acknowledgements}

This study was supported by the National Natural Science Foundation of China (No. 11575274), Strategic Priority Research Program of the Chinese Academy of Sciences (No. XDB16010600), and Ministry of Science and Technology of the People's Republic of China (Nos. 2016YFA0401102 and 2018YFA0404803).

\section{References}

1. G. A. Mourou, T. Tajima, and S. V. Bulanov, Rev. Mod. Phys. 78, 309 (2006).

2. A. Macchi, M. Borghesi, and M. Passoni, Rev. Mod. Phys. 85, 751 (2013).

3. H. Daido, M. Nishiuchi, and A. S. Pirozhkov, Phys. Soc. 75, 056401 (2012).

4. J. Schreiber, P. R. Bolton, and K. Parodi, Rev. Sci. Instrum. 87, 071101 (2016).

5. M. Tabak, J. Hammer, M. E. Glinsky, W. L. Kruer, S. C. Wilks, J. Woodworth, E. M. Campbell, M. D. Perry, and R. J. Mason, Phys. Plasmas 1, 1626 (1994).

6. N. Naumova, T. Schlegel, V. T. Tikhonchuk, C. Labaune, I. V. Sokolov, and G. Mourou, Phys. Rev. Lett. 102, 025002 (2009).

7. S. V. Bulanov, T. Z. Esirkepov, V. S. Khoroshkov, A. V. Kuznetsov, and F. Pegoraro, Phys. Lett. A 299, 240 (2002).

8. S. V. Bulanov and V. S. Khoroshkov, Plasma Phys. Rep. 28, 453 (2002).

9. S. V. Bulanov, J. J. Wilkens, T. Z. Esirkepov, G. Korn, G. Kraft, S. D. Kraft, M. Molls, and V. S. Khoroshkov, Phys.Usp. 57, 1149 (2014).

10. M. Borghesi, D. H. Campbell, A. Schiavi, M. G. Haines, O. Willi, A. J. MacKinnon, P. Patel, L. A. Gizzi, M. Galimberti, R. J. Clarke, F. Pegoraro, H. Ruhl, and S. Bulanov, Phys. Plasmas 9, 2214 (2002). 
11. S. Kar, A. Green, H. Ahmed, A. Alejo, A. P. L. Robinson, M. Cerchez, R. Clarke, D. Doria, S. Dorkings, J. Fernandez, S. R. Mirfayzi, P. McKenna, K. Naughton, D. Neely, P. Norreys, C. Peth, H. Powell, J. A. Ruiz, J. Swain, O. Willi, and M. Borghesi, New J. Phys. 18, 053002 (2016).

12. M. Roth, D. Jung, K. Falk, N. Guler, O. Deppert, M. Devlin, A. Favalli, J. Fernandez, D. Gautier, M. Geissel, R. Haight, C. E. Hamilton, B. M. Hegelich, R. P. Johnson, F. Merrill, G. Schaumann, K. Schoenberg, M. Schollmeier, T. Shimada, T. Taddeucci, J. L. Tybo, F. Wagner, S. A. Wender, C. H. Wilde, and G. A. Wurden, Phys. Rev. Lett. 110, 044802 (2013).

13. K. W. D. Ledingham, P. McKenna, and R. P. Singhal, Science 300, 1107 (2003).

14. N. V. Zamfir, Eur. Phys. J. 223, 1221 (2014).

15. E. L. Clark, R. Allott, F. N. Beg, C. N. Danson, A. Machacek, V. Malka, Z. Najmudin, D. Neely, P. A. Norreys, M. R. Salvati, M. I. K. Santala, M. Tatarakis, I. Watts, M. Zepf, and A. E. Dangor, IEEE Trans. Plasma Sci. 28, 1184 (2000).

16. A. Macchi, F. Cattani, T. V. Liseykina, and F. Cornolti, Phys. Rev. Lett. 94, 165003 (2005).

17. X. Zhang, B. Shen, X. Li, Z. Jin, F. Wang, and M. Wen, Phys. Plasmas 14, 123108 (2007).

18. X. Q. Yan, C. Lin, Z. M. Sheng, Z. Y. Guo, B. C. Liu, Y. R. Lu, J. X. Fang, and J. E. Chen, Phys. Rev. Lett. 100, 135003 (2008).

19. A. P. L. Robinson, M. Zepf, S. Kar, R. G. Evans, and C. Bellei, New J. Phys. 10, 013021 (2008).

20. O. Klimo, J. Psikal, J. Limpouch, and V. T. Tikhonchuk, Phys. Rev. ST Accel. Beams 11, 031301 (2008).

21. B. Qiao, M. Zepf, M. Borghesi, and M. Geissler, Phys. Rev. Lett. 102, 145002 (2009).

22. A. Henig, S. Steinke, M. Schnürer, T. Sokollik, R. Hörlein, D. Kiefer, D. Jung, J. Schreiber, B. M. Hegelich, X. Q. Yan, J. Meyer-ter-Vehn, T. Tajima, P. V. Nickles, W. Sandner, and D. Habs, Phys. Rev. Lett. 103, 245003 (2009).

23. S. V. Bulanov, E. Y. Echkina, T. Z. Esirkepov, I. N. Inovenkov, M. Kando, F. Pegoraro, and G. Korn, Phys. Rev. Lett. 104, 135003 (2010).

24. W. P. Wang, B. F. Shen, X. M. Zhang, L. L. Ji, M. Wen, J. C. Xu, Y. H. Yu, Y. L. Li, and Z. Z. Xu, Phys. Plasmas 18, 013103 (2011).

25. T.-P. Yu, A. Pukhov, G. Shvets, and M. Chen, Phys. Rev. Lett. 105, 065002 (2010).

26. J. H. Bin, W. J. Ma, H. Y. Wang, M. J. V. Streeter, C. Kreuzer, D. Kiefer, M. Yeung, S. Cousens, P. S. Foster, B. Dromey, X. Q. Yan, R. Ramis, J. Meyer-ter-Vehn, M. Zepf, and J. Schreiber, Phys. Rev. Lett. 115, 064801 (2015).

27. Y. Wan, C. H. Pai, C. J. Zhang, F. Li, Y. P. Wu, J. F. Hua, W. Lu, Y. Q. Gu, L. O. Silva, C. Joshi, and W. B. Mori, Phys. Rev. Lett. 117, 234801 (2016).

28. X. F. Shen, B. Qiao, H. Zhang, S. Kar, C. T. Zhou, H. X. Chang, M. Borghesi, and X. T. He, Phys. Rev. Lett. 118, 204802 (2017)

29. C. Scullion, D. Doria, L. Romagnani, A. Sgattoni, K. Naughton, D. R. Symes, P. McKenna, A. Macchi, M. Zepf, S. Kar, and M. Borghesi, Phys. Rev. Lett. 119, 054801 (2017).

30. A. Higginson, R. J. Gray, M. King, R. J. Dance, S. D. R. Williamson, N. M. H. Butler, R. Wilson, R. Capdessus, C. Armstrong, J. S. Green, S. J. Hawkes, P. Martin, W. Q. Wei, S. R. Mirfayzi, X. H. Yuan, S. Kar, M. Borghesi, R. J. Clarke, D. Neely, and P. McKenna, Nat. Commun. 9, 724 (2018).

31. A. P. L. Robinson, P. Gibbon, M. Zepf, S. Kar, R. G. Evans, and C. Bellei, Plasma Phys. Control. Fusion 51, 024004 (2009).

32. W. P. Wang, B. F. Shen, and Z. Z. Xu, Phys. Plasmas 24, 013104 (2017).
33. F. Pegoraro and S. V. Bulanov, Phys. Rev. Lett. 99, 065002 (2007).

34. C. A. J. Palmer, J. Schreiber, S. R. Nagel, N. P. Dover, C. Bellei, F. N. Beg, S. Bott, R. J. Clarke, A. E. Dangor, S. M. Hassan, P. Hilz, D. Jung, S. Kneip, S. P. D. Mangles, K. L. Lancaster, A. Rehman, A. P. L. Robinson, C. Spindloe, J. Szerypo, M. Tatarakis, M. Yeung, M. Zepf, and Z. Najmudin, Phys. Rev. Lett. 108, 225002 (2012).

35. B. Eliasson, New J. Phys. 17, 033026 (2015).

36. A. Sgattoni, S. Sinigardi, L. Fedeli, F. Pegoraro, and A. Macchi, Phys. Rev. E 91, 013106 (2015).

37. X. Q. Yan, H. C. Wu, Z. M. Sheng, J. E. Chen, and J. MeyerTer-Vehn, Phys. Rev. Lett. 103, 135001 (2009).

38. X. Zhang, B. Shen, L. Ji, W. Wang, J. Xu, Y. Yu, and X. Wang, Phys. Plasmas 18, 073101 (2011).

39. L. Ji, B. Shen, X. Zhang, F. Wang, Z. Jin, X. Li, M. Wen, and J. R. Cary, Phys. Rev. Lett. 101, 164802 (2008).

40. X. Zhang, B. Shen, L. Ji, F. Wang, Z. Jin, X. Li, M. Wen, and J. R. Cary, Phys. Rev. ST Accel. Beams 12, 021301 (2009).

41. W. P. Wang, X. M. Zhang, X. F. Wang, X. Y. Zhao, J. C. Xu, Y. H. Yu, L. Q. Yi, Y. Shi, L. G. Zhang, T. J. Xu, C. Liu, Z. K. Pei, and B. F. Shen, High Power Laser Sci. Eng. 2, e9 (2014).

42. W. P. Wang, B. F. Shen, X. M. Zhang, L. L. Ji, Y. H. Yu, L. Q. Yi, X. F. Wang, and Z. Z. Xu, Phys. Rev. ST Accel. Beams 15, 081302 (2012).

43. B. Rus, F. Batysta, J. Čáp, M. Divoký, M. Fibrich, M. Griffiths, R. Haley, T. Havlíček, M. Hlavác, J. Hřebíček, P. Homer, P. Hříbek, J. Jand'ourek, L. Juha, G. Korn, P. Korouš, M. Košelja, M. Kozlová, D. Kramer, M. Krůs, J. C. Lagron, J. Limpouch, L. MacFarlane, M. Malý, D. Margarone, P. Matlas, L. Mindl, J. Moravec, T. Mocek, J. Nejdl, J. Novák, V. Olšovcová, M. Palatka, J. P. Perin, M. Pešlo, J. Polan, J. Prokůpek, J. Řídký, K. Rohlena, V. Růžička, M. Sawicka, L. Scholzová, D. Snopek, P. Strkula, and L. Švéda, Proc. SPIE 8080, 808010 (2011)

44. N. V. Zamfir, J. Phys. Conf. Ser. 366, 012052 (2012).

45. J. P. Zou, C. L. Blanc, D. N. Papadopoulos, G. Ch́eriaux, P. Georges, G. Mennerat, F. Druon, L. Lecherbourg, A. Pellegrina, P. Ramirez, F. Giambruno, A. Fŕeneaux, F. Leconte, D. Badarau, J. M. Boudenne, D. Fournet, T. Valloton, J. L. Paillard, J. L. Veray, M. Pina, P. Monot, J. P. Chambaret, P. Martin, F. Mathieu, P. Audebert, and F. Amiranoff, High. Power Laser Sci. Eng. 3, e2 (2015).

46. A. V. Bashinov, A. A. Gonoskov, A. V. Kim, G. Mourou, and A. M. Sergeev, Eur. Phys. J. Spec. Top. 223, 1105 (2014).

47. I. Prencipe, J. Fuchs, S. Pascarelli, D. W. Schumacher, R. B. Stephens, N. B. Alexander, R. Briggs, M. Büscher, M. O. Cernaianu, A. Choukourov, M. De Marco, A. Erbe, J. Fassbender, G. Fiquet, P. Fitzsimmons, C. Gheorghiu, J. Hund, L. G. Huang, M. Harmand, N. J. Hartley, A. Irman, T. Kluge, Z. Konopkova, S. Kraft, D. Kraus, V. Leca, D. Margarone, J. Metzkes, K. Nagai, W. Nazarov, P. Lutoslawski, D. Papp, M. Passoni, A. Pelka, J. P. Perin, J. Schulz, M. Smid, C. Spindloe, S. Steinke, R. Torchio, C. Vass, T. Wiste, R. Zaffino, K. Zeil, T. Tschentscher, U. Schramm, and T. E. Cowan, High Power Laser Sci. Eng. 5, e17 (2017).

48. O. Shavit, Y. Ferber, J. Papeer, E. Schleifer, M. Botton, A. Zigler, and Z. Henis, High Power Laser Sci. Eng. 6, e7 (2018).

49. S. Weng, Q. Zhao, Z. Sheng, W. Yu, S. Luan, M. Chen, L. Yu, M. Murakami, W. B. Mori, and J. Zhang, Optica 4, 1086 (2017).

50. H. Y. Wang, C. Lin, Z. M. Sheng, B. Liu, S. Zhao, Z. Y. Guo, Y. R. Lu, X. T. He, J. E. Chen, and X. Q. Yan, Phys. Rev. Lett. 107, 265002 (2011).

51. L. L. Ji, B. F. Shen, X. M. Zhang, F. C. Wang, Z. Y. Jin, C. Q. Xia, M. Wen, W. P. Wang, J. C. Xu, and M. Y. Yu, Phys. Rev. Lett. 103, 215005 (2009). 